\title{
Intracranial Arteriovenous Shunting: Detection with Arterial Spin-Labeling and Susceptibility-Weighted Imaging Combined
}

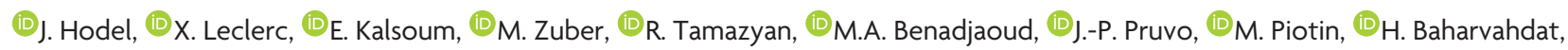
(1) M. Zins, and ${ }^{-}$R. Blanc

\begin{abstract}
BACKGROUND AND PURPOSE: Arterial spin-labeling and susceptibility-weighted imaging are 2 MR imaging techniques that do not require gadolinium. The study aimed to assess the accuracy of arterial spin-labeling and SWI combined for detecting intracranial arteriovenous shunting in comparison with conventional MR imaging.
\end{abstract}

MATERIALS AND METHODS: Ninety-two consecutive patients with a known $(n=24)$ or suspected arteriovenous shunting $(n=68)$ underwent digital subtraction angiography and brain MR imaging, including arterial spin-labeling/SWI and conventional angiographic MR imaging (3D TOF, 4D time-resolved, and 3D contrast-enhanced MRA). Arterial spin-labeling/SWI and conventional MR imaging were reviewed separately in a randomized order by 2 blinded radiologists who judged the presence or absence of arteriovenous shunting. The accuracy of arterial spin-labeling/SWI for the detection of arteriovenous shunting was calculated by using the area under receiver operating curve with DSA as reference standard. $\kappa$ coefficients were computed to determine interobserver and intermodality agreement.

RESULTS: Of the 92 patients, DSA showed arteriovenous shunting in 63 (arteriovenous malformation in 53 and dural arteriovenous fistula in 10). Interobserver agreement was excellent $(\kappa=0.83-0.95)$. In 5 patients, arterial spin-labeling/SWI correctly detected arteriovenous shunting, while the conventional angiographic MR imaging did not. Compared with conventional MR imaging, arterial spin-labeling/SWI was significantly more sensitive ( 0.98 versus $0.90, P=.04$ ) and equally specific $(0.97)$ and showed significantly higher agreement with DSA $(\kappa=0.95$ versus $0.84, P=.01)$ and higher area under the receiver operating curve $(0.97$ versus $0.93, P=.02)$.

CONCLUSIONS: Our study showed that the combined use of arterial spin-labeling and SWI may be an alternative to contrast-enhanced MRA for the detection of intracranial arteriovenous shunting.

ABBREVIATIONS: $A S L=$ arterial spin-labeling; $\mathrm{AUC}=$ area under the receiver operating curve; $\mathrm{AVS}=$ arteriovenous shunting; $\mathrm{DAVF}=$ dural arteriovenous fistula; $\mathrm{ICH}=$ intracranial hemorrhage

ntracranial arteriovenous shunting (AVS) related to dural arteriovenous fistulas (DAVFs) or AVMs may lead to several neurologic complications, including acute intracranial hemorrhage $(\mathrm{ICH}) .{ }^{1-3}$ DSA remains the reference standard to confirm AVS and assess its angioarchitecture. However, conventional brain MR imaging, including time-of-flight and contrast-enhanced MR angiography, is commonly performed in patients with suspected

Received May 13, 2016; accepted after revision August 16.

From the Departments of Radiology (J.H., M.Z.) and Neurology (M.Z., R.T.), Saint Joseph Hospital, Paris, France; Department of Neuroradiology (J.H., E.K.), CentreHospitalier-Universitaire Henri Mondor, Créteil, France; Department of Neuroradiology (X.L., J.-P. P.), Roger Salengro Hospital, Lille, France; Department of Radiobiology and Epidemiology (M.A.B.), Institute for Radiological Protection and Nuclear Safety (IRSN), Fontenay-Aux-Roses, France; and Department of Interventional Neuroradiology (M.P., H.B., R.B.), Rothschild Foundation Hospital, Paris, France.

J. Hodel and X. Leclerc are co-first authors.

Please address correspondence to Jérôme Hodel, MD, PhD, Department of Neuroradiology, CHU Henri Mondor, Créteil, France; e-mail: jerome.hodel@gmail.com

http://dx.doi.org/10.3174/ajnr.A4961
AVS, particularly in the setting of acute ICH. Time-resolved (4D) contrast-enhanced MRA is routinely performed in patients suspected of having AVS or for characterizing a known AVM or DAVF. ${ }^{4-6}$ Limitations of this approach include low spatial resolution, incomplete brain coverage, and technical difficulties. ${ }^{4-7}$

Recently, 2 noncontrast MR imaging techniques, namely SWI and arterial spin-labeling (ASL), were also reported useful for the detection of intracranial AVS. SWI can demonstrate the venous drainage as high signal intensity because of increased blood flow and the presence of a large amount of oxyhemoglobin, ${ }^{8-11}$ while ASL can improve the detection of AVS by showing venous ASL signal. ${ }^{12-16}$ To our knowledge, no previous study has yet compared the accuracy for detecting AVS of these noncontrast techniques with the conventional MR imaging protocol, including contrast-enhanced MRA. During a 4-year period, we have systematically performed 3T MR imaging, including SWI, ASL, and conventional angiographic MR imaging (ie, TOF-MRA, 3D and 4D contrast-enhanced MRA), in all consecutive patients referred 


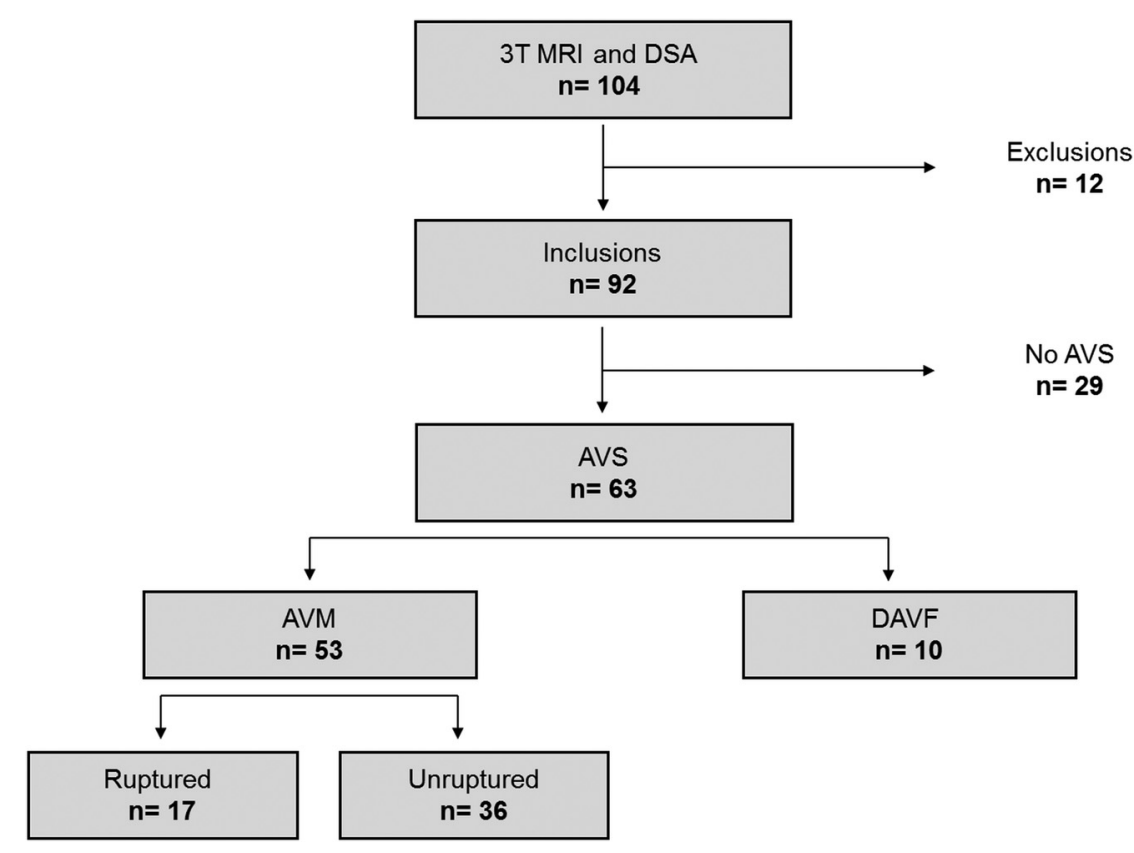

FIG 1. Flow chart illustrating patient selection.

for DSA (considered the reference standard in the present study) for known or suspected AVS. This study sought to determine the accuracy of the combined use of ASL and SWI (ASL/SWI) for the detection of AVS in comparison with conventional MR imaging, including contrast-enhanced MRA.

\section{MATERIALS AND METHODS \\ Patients and Brain Imaging}

This study was approved by the Rothschild Foundation Hospital institutional review board, and written informed consent was obtained from all subjects. From September 1, 2011, to August 31, 2015, 104 consecutive patients ( 45 females and 59 males; mean age, 48.3 years; range, $11-81$ years) underwent DSA for a known $(n=26)$ or suspected $(n=78)$ AVS. Known AVS included brain AVM previously treated by embolization and/or radiosurgery. In this patient group, DSA was performed to evaluate nidus reduction or detect a potential residual shunting. DAVF or brain AVM was suspected in patients with brain hematoma (according to clinical status, patient age, medical history, and location of hemorrhage), pulsatile tinnitus, and suggestive findings on brain MR imaging/CT. In these patients, DSA was performed to confirm AVS and to analyze its location and angioarchitecture.

DSA was performed on a flat panel biplane system (Allura; Philips Healthcare, Best, the Netherlands) and included a selective injection in both the internal and external carotid arteries and vertebral arteries with at least 2 intracranial views (frontal and sagittal). When necessary, 3D rotational angiography with MIP reconstructions and additional oblique views was performed. Each angiogram was acquired at 2 frames per second with a $1024 \times 1024$ matrix size and a $20-\mathrm{cm}$ or $27-\mathrm{cm}$ FOV.

In all 104 consecutive patients, we systematically performed 3T MR imaging (Discovery MR750; GE Healthcare, Milwaukee, Wisconsin) once per patient as required by the institutional review board and within the 3 weeks following DSA. Patients were excluded from the study on the basis of at least 1 of the following conditions: 1) The time interval between MR imaging and DSA was $>4$ weeks; 2 ) an endovascular or a radiosurgical procedure was performed in the interval between MR imaging and DSA; and 3) image artifacts prevented accurate analysis of the MR images. Among the 104 consecutive patients, 12 were excluded from the study because of major artifacts on MR imaging. Finally, the study group consisted of 92 patients ( 40 females and 52 males; mean age, 47.4 years; range, 11-81 years) with a mean time interval between MR imaging and DSA of 13 days (range, 2-21 days). A flow chart illustrating the patient characteristics is shown in Fig 1.

The conventional MR imaging protocol included 2D diffusion-weighted imaging, 2D gradient-echo $\mathrm{T}^{*}, 3 \mathrm{D}$ FLAIR, 3D TOF MRA, 4D contrast-enhanced MRA (TR, $2.6 \mathrm{~ms}$; TE, $1.1 \mathrm{~ms}$; temporal resolution, 1 second; voxel size, $1 \times 1 \times 1 \mathrm{~mm}$ ) directly followed by a 3D high-resolution contrast-enhanced MRA (TR, $3.6 \mathrm{~ms}$; TE, $1.5 \mathrm{~ms}$; voxel size, $0.6 \times 0.6 \times 0.6 \mathrm{~mm})$, and postcontrast $3 \mathrm{D}$ gradient-echo $\mathrm{T} 1 \mathrm{WI}$ sequences (voxel size, $1 \times 1 \times 1 \mathrm{~mm}$ ). In addition, SWI (TR, 40 $\mathrm{ms}$; TE, $25 \mathrm{~ms}$; voxel size, $1 \times 1 \times 2 \mathrm{~mm}$ ) and ASL (3D FSE; pseudocontinuous; postlabel delay, $1525 \mathrm{~ms}$; section thickness, $4 \mathrm{~mm}$ ) were performed in each subject before gadolinium administration.

4D contrast-enhanced MRA covered a half-brain that was chosen according to DSA results to optimize temporal and spatial resolution. Intravenous gadobutrol (Gadovist; Bayer Schering Pharma, Berlin, Germany) was administered at a concentration of $0.1 \mathrm{mmol} / \mathrm{kg}$ during the acquisition of $4 \mathrm{D}$ contrast-enhanced MRA.

\section{Image Analysis}

DSA examinations were interpreted by 2 interventional neuroradiologists (X.L., J.-P.P.) together, with $>20$ years of experience. They reviewed the DSA images of all 92 patients to determine the presence or absence of AVS. In case of AVS, they assigned the vascular malformation as DAVF or AVM. A lesion was considered consistent with a DAVF if meningeal arterial feeders and a fistulous point on the dura mater were identified. A lesion was considered consistent with AVM if arterial feeders and a complex AVS (nidus) located in the brain were identified. Developmental venous anomaly was also located by using DSA, if present.

MR images were first anonymized and randomly mixed. Two experienced neuroradiologists with 14 (R.B.) and 10 (J.H.) years of experience, blinded to the results of DSA and clinical data, independently reviewed all the MR imaging datasets for the presence of AVS. Four independent readings took place 2 weeks apart to avoid recall bias with the following sequences available: 1) ASL alone, 2) SWI alone, 3) ASL/SWI, and 4) the "conventional MR 
imaging protocol," including diffusion-weighted imaging, T2*, 3D FLAIR, TOF MRA, 4D and 3D contrast-enhanced MRA, and postcontrast 3D T1WI MR imaging. Criteria for the presence of AVS on MR images were defined as follows:

With ASL, the presence of an intracranial venous hypersignal within the dural sinuses or cortical veins.

With SWI, a hyperintense venous structure and/or direct visualization of the nidus/fistulous point.

With $4 \mathrm{D}$ contrast-enhanced MRA, visualization of the venous drainage at the arterial phase.

With TOF and 3D contrast-enhanced MRA, enlarged and dilated serpiginous vessels and/or direct visualization of the fistulous point/nidus.

For each patient, all the MR images were assessed by using a dedicated workstation (Advantage Windows Workstation; GE Healthcare) with multiplanar reformations available. With SWI, axial 10 -mm-thick maximum intensity projections were systematically reconstructed as well as raw data and CBF color maps of ASL. With 4D contrast-enhanced MRA, a time-resolved MIP sagittal view was available as well as each of the 3D MRA volumes generated. When assessing combined MR images, the readers had the ability to merge MR images from different sequences, with or without manual thresholding, by using the available fusion software (3D synchroview, Advantage Windows Workstation; GE Healthcare). Discrepancies between readers were resolved by con-

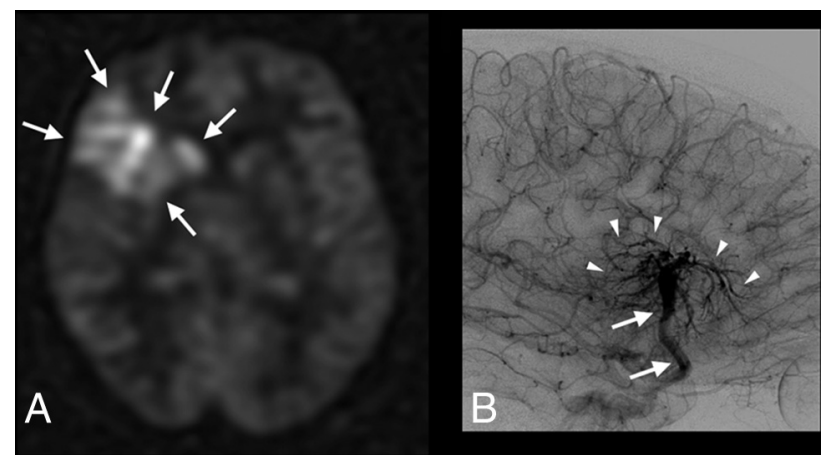

FIG 2. Patient 62 with a complex developmental venous anomaly. ASL raw data $(A)$ and right internal carotid artery angiogram, venous phase, lateral view (B). Increased signal-intensity is visible on ASL images within the right frontal lobe and deep brain nuclei ( $A$, arrows). ASL images were considered suggestive of AVS by 1 blinded reader. Developmental venous anomaly was correctly diagnosed (and thus absence of AVS) by using SWI and a combination of SWI and ASL (not shown). DSA confirms the diagnosis of developmental venous anomaly by revealing a classic umbrella-shaped aspect at the venous phase with medullary veins ( $B$, arrowheads) draining into an enlarged collector ( $B$, arrows), which further drains into the superficial Sylvian vein and cavernous sinus.

Table 1: Diagnosis of AVS (number of patients correctly diagnosed) using ASL, SWI, and conventional MRI

\begin{tabular}{lcccc}
\hline & \multicolumn{4}{c}{ DSA } \\
\cline { 2 - 5 } \multicolumn{1}{c}{ MRI Sequences } & No AVS $(\boldsymbol{n}=29)$ & AVS $(\boldsymbol{n}=63)$ & DAVF $(\boldsymbol{n}=10)$ & AVM $(\boldsymbol{n}=53)$ \\
\hline SWI & $28(97 \%)$ & $55(87 \%)$ & $8(80 \%)$ & $47(89 \%)$ \\
ASL & $26(90 \%)$ & $60(95 \%)$ & $8(80 \%)$ & $52(98 \%)$ \\
ASL/SWI & $28(97 \%)$ & $62(98 \%)$ & $9(90 \%)$ & $53(100 \%)$ \\
Conventional MRI & $28(97 \%)$ & $57(90 \%)$ & $9(90 \%)$ & $48(91 \%)$ \\
\hline
\end{tabular}

Note:-ASL/SWI indicates ASL and SWI combined; conventional MRI, conventional MRI protocol (including diffusionweighted imaging, gradient-echo T2*, 3D FLAIR, TOF MRA, 4D and 3D contrast-enhanced MRA, and postcontrast 3D TTWI sequences). sensus by using a panel, including an additional board-certified neuroradiologist (X.L.). In each patient, signal abnormalities previously detected on MR images by the blinded readers were correlated with the presence or absence of AVS on DSA. Medical records were also systematically reviewed for acute ICH and previous surgical or endovascular AVS treatments.

\section{Statistical Analysis}

Statistical analysis was performed by using SAS software, Version 9.3 (SAS Institute, Cary, North Carolina). Interobserver and intermodality agreement was calculated by using the Cohen $\kappa$ test. $\kappa$ values were interpreted as follows: $\kappa$ value of 0 indicates poor agreement; $\kappa$ values of $0.01-0.20$ indicate minor agreement; $\kappa$ values of $0.21-0.40$, fair agreement; $\kappa$ values of $0.41-0.60$, moderate agreement; $\kappa$ values of $0.61-0.80$, good agreement; and $\kappa$ values of $0.81-1$, excellent agreement. For each of the 4 independent readings, we calculated standard diagnostic accuracy parameters (ie, sensitivity, specificity, and positive and negative predictive values) for the detection of AVS in the whole cohort $(n=92)$, by using DSA as a reference standard. Diagnostic accuracy was also determined by calculating the area under the receiver operating curve (AUC), which equals 0.5 and 1 for models with random and perfect discrimination, respectively. In addition, sensitivity and specificity were calculated in the 2 subgroups: the patients with ICH $(n=24)$ and those previously treated for AVS $(n=24)$. The McNemar test was used to compare measures of diagnostic accuracy. The confidence intervals were calculated by using normal based approximation and were truncated to avoid values of $>1$.

\section{RESULTS}

\section{DSA Findings}

Among the 92 included patients, 68 underwent DSA for a suspected AVS, and 24, for a known AVS previously treated (including 14 without AVS on DSA and 10 with angiographically proved residual AVS related to an AVM). Of these 92 patients, DSA was consistent with AVS in 63 patients (68.5\%; 23 females, 40 males; mean age, 45.8 years; range, $11-81$ years), including DAVFs in 10 and AVMs in 53. DAVFs were confined to the sinus with antegrade flow (type I according to the Cognard classification) in 2 patients; confined to the sinus with reflux into the sinus (type IIa) in 1 patient; with reflux into the cortical veins (type IIb) in 1 patient; directly draining into the cortical veins (type III) in 4 patients; and with venous ectasia (type IV) in 2 patients. In the 53 patients with AVMs, the Spetzler-Martin classification ranged from I to $\mathrm{V}$ (grade I in 14, grade II in 13, grade III in 15 , grade IV in 8 , and grade $V$ in 3 ); and the nidus size was $<3$ $\mathrm{cm}$ in 37 (including 13 patients with a nidus size $\leq 1 \mathrm{~cm}$ ), between 3 and $6 \mathrm{~cm}$ in 13 , and $>6 \mathrm{~cm}$ in 3 .

The remaining 29 patients without AVS (17 females; mean age, 45.8 years; range, 11-81 years) consisted of 7 patients with no lesion detected by DSA, 14 with a previously treated and completely cured AVS (AVM in 8 and DAVF in 6), and 8 with developmental venous anomalies. In 24 patients with acute 
Table 2: Diagnostic accuracy parameters using ASL, SWI, and conventional MRI

\begin{tabular}{|c|c|c|c|c|c|}
\hline \multirow[b]{2}{*}{ MRI Sequences } & \multicolumn{5}{|c|}{ Diagnostic Accuracy Parameters } \\
\hline & Se $(95 \% \mathrm{Cl})$ & $\mathrm{Sp}(95 \% \mathrm{Cl})$ & PPV $(95 \% \mathrm{Cl})$ & NPV $(95 \% \mathrm{Cl})$ & AUC $(95 \% \mathrm{Cl})$ \\
\hline SWI & $0.87(0.79-0.96)$ & $0.97(0.90-1)$ & $0.98(0.90-1)$ & $0.78(0.61-0.90)$ & $0.92(0.85-0.96)$ \\
\hline ASL & $0.95(0.90-1)$ & $0.90(0.78-1)$ & $0.95(0.87-0.99)$ & $0.90(0.73-0.98)$ & $0.92(0.84-0.97)$ \\
\hline ASL/SWI & $0.98(0.95-1)$ & $0.97(0.90-1)$ & $0.98(0.95-1)$ & $0.97(0.90-1)$ & $0.97(0.90-1)$ \\
\hline Conventional MRI & $0.90(0.83-0.98)$ & $0.97(0.90-1)$ & $0.98(0.91-1)$ & $0.92(0.65-0.93)$ & $0.93(0.87-0.97)$ \\
\hline
\end{tabular}

Note:-Se indicates sensitivity; Sp, specificity; PPV, predictive positive value; NPV, negative predictive value.

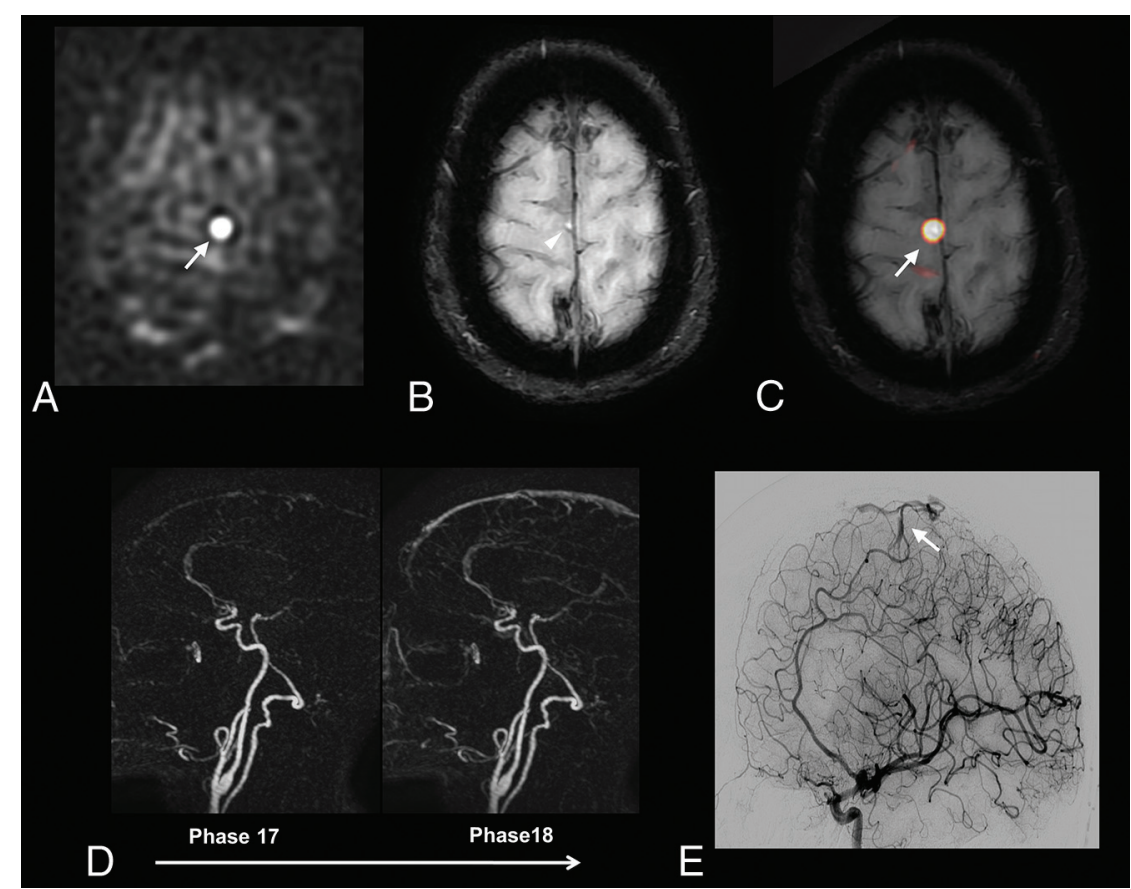

FIG 3. A 60-year-old patient with a right paracentral AVM. ASL raw data $(A)$ demonstrates a strong hypersignal at the anterior part of the right paracentral region ( $A$, arrow). The slight venous hypersignal related to AVS was initially missed by the blinded readers by using SWI alone (B, arrowhead) but was correctly identified by using ASL and SWI combined (C, ASL) SWI merged image, arrow). Findings of time-resolved 4D contrast-enhanced MRA (D) were considered negative by the blinded readers. DSA reveals a small pial AVM in the right paracentral region (E, arrow).

$\mathrm{ICH}$, DSA revealed AVS in 17, including 7 with AVMs under treatment and 10 with an untreated AVM.

\section{Interobserver Agreement}

Interobserver agreement ( $\kappa$ value, 95\% CI) between both blinded readers was the following: $0.83(0.78-0.98)$ for SWI; $0.91(0.82-1)$ for conventional MR imaging protocol; and 0.95 (0.88-1) for ASL and ASL/SWI. There were 2 disagreements with ASL: 1 hyperintensity judged as too faint by 1 reader to be related to AVS and 1 other suggestive of developmental venous anomaly for reader 1 and AVS according to reader 2 (Fig 2). Disagreements between readers were observed in 5 cases with SWI.

\section{Detection of AVS by Using MR Imaging}

Results are summarized in Tables 1 and 2. ASL and SWI used in combination correctly demonstrated AVS in 5 patients in whom the conventional MR imaging protocol had failed (Figs 3-5). These patients presented with small AVMs, with a nidus size of $<1 \mathrm{~cm}$, associated with acute ICH in 1 subject and previously treated in another.

To detect AVS, ASL/SWI was significantly more sensitive than
( 0.98 versus $0.90, P=.04)$ and as specific as $(0.97)$ the conventional MR imaging protocol and had a significantly higher agreement with DSA $(\kappa=0.95$ versus $0.83, P=.01)$ and higher AUC (0.97 versus $0.93, P=.02$ ). Considering the agreement with DSA or AUC, ASL and SWI did not differ significantly $(\kappa=$ 0.85 versus $0.79, P=.5$, and $\mathrm{AUC}=$ $0.92, P=.9$, respectively).

Within the subgroups of patients with ICH $(n=24)$ and those with a previously treated AVS $(n=24)$, ASL/SWI was the most sensitive approach for detecting AVS. In patients with ICH, ASL/ SWI was $100 \%$ sensitive and $86 \%$ specific, while conventional MR imaging protocol was $82 \%$ sensitive and $86 \%$ specific. In patients previously treated, ASL/SWI was $100 \%$ sensitive and specific, while the conventional MR imaging protocol was 90\% sensitive and $100 \%$ specific.

\section{DISCUSSION}

Our study showed that 2 noncontrast MR imaging techniques used in combination, namely ASL and SWI, may offer a noninvasive and practical alternative to contrast-enhanced MRA for the detection of intracranial AVS. Such a finding is of clinical relevance if one considers the risk of nephrogenic systemic fibrosis in patients with renal insufficiency. ${ }^{17}$ Other limitations of the use of gadolinium chelate are related to age, pregnancy or lactation, and the recent data regarding gadolinium retention in deep brain nuclei. ${ }^{18,19}$ Indeed, patients with AVM or DAVF frequently undergo repeated MR imaging examinations during their follow-up to rule out residual AVS.

In the present study, we assumed that the combined use of ASL and SWI (ASL/SWI) could be as successful as contrast-enhanced MR imaging to detect intracranial AVS. This explains why we separately interpreted contrast-enhanced MR images and ASL/ SWI. The excellent negative predictive value of ASL/SWI suggests that such an approach may be of use in ruling out AVS in patients with acute ICH and/or equivocal conventional MR imaging findings, potentially sparing them from invasive DSA evaluation. Conversely, considering the high positive predictive value of ASL/ SWI, DSA should also be performed in patients with suggestive signal abnormalities to confirm and characterize the AVS. Several 


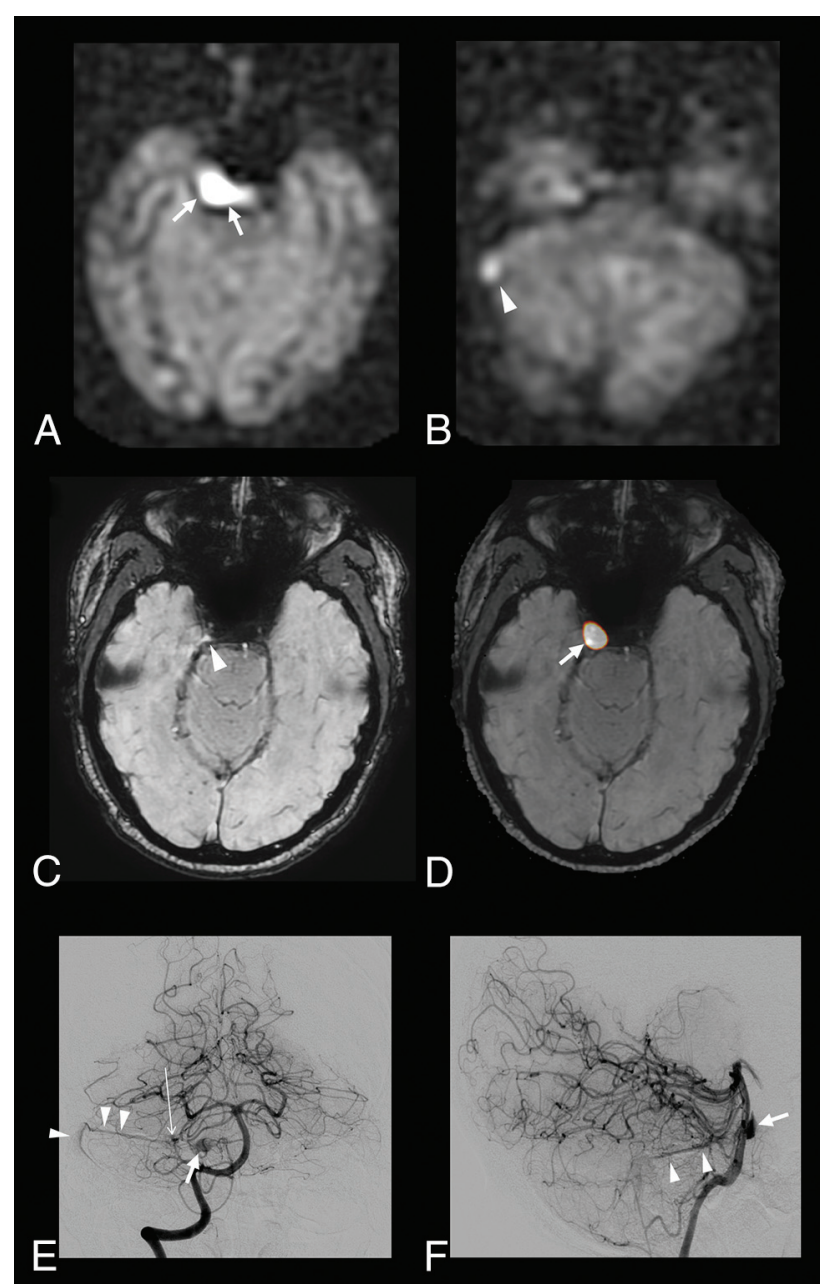

FIG 4. A 52-year-old patient with a right cerebellar AVM draining into the right transverse sinus and the right periclival plexus. ASL raw data demonstrate a strong hypersignal within the right periclival plexus ( $A$, arrows) and the right lateral sinus ( $B$, arrowhead). With SWI alone, the slight venous hypersignal within the right periclival plexus and related to AVS was initially missed by the blinded readers ( $C$, arrowhead) but correctly identified by using ASL and SWI combined (D, ASL/SWI merged image, arrow). Findings of time-resolved and high-resolution contrast-enhanced MRA were negative. Anteroposterior $(E)$ and lateral $(F)$ views from the right vertebral conventional angiograms show a small nidus ( $E$, long arrow) draining into the right lateral sinus through the right superior petrosal sinus ( $E$ and $F$, arrowheads) and into the periclival plexus through a lateropontine vein ( $E$ and $F$, arrow).

advantages of the combined use of SWI and ASL may be stressed. First, SWI and ASL provide full-brain coverage, while 4D contrast-enhanced MRA is frequently performed with half-brain coverage to optimize spatial and temporal resolution. Second, the detection of AVS on SWI and ASL images is simple (with a strong hypersignal related to AVS, easily recognizable from the background) and reproducible as suggested by the excellent interobserver agreement, while imaging findings on postcontrast MR imaging may be less specific because dilated intracranial veins may be found in a wide range of conditions.

Our results were in agreement with previous studies showing that SWI and ASL are 2 effective techniques to detect intracranial AVS. ${ }^{8-15}$ Jagadeesan et al $^{10}$ demonstrated that SWI was $93 \%$ sensitive and $98 \%$ specific for the detection of AVS in patients with AVM. SWI was also found to accurately differentiate between

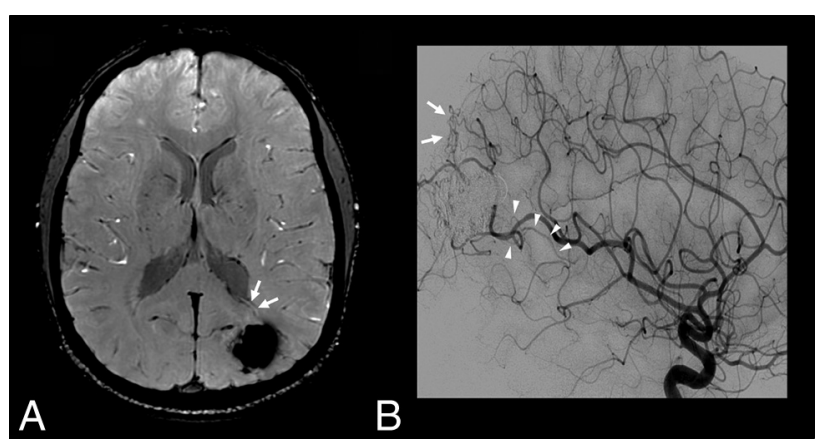

FIG 5. Patient 26 with a previously treated AVM and residual AVS according to DSA. SWI (A) and left internal carotid artery angiogram, arterial phase, lateral view (B). An increased signal intensity is visible with SWI within a deep vein adjacent to the treated nidus ( $A$, arrows), suggestive of AVS. Of note, findings of ASL and contrast-enhanced MR images were considered negative (not shown). DSA confirms the presence of dysplastic vessels around the cast of the embolic agent ( $B$, arrows) and an early opacification of venous drainage ( $B$, arrowheads) coursing toward the deep venous system at the anterior and inferior pole of the embolized AVM.

high-flow and low-flow abnormalities of the vein of Galen in children. ${ }^{9}$ The high sensitivity of ASL for the diagnosis of AVS was also previously reported. ${ }^{13,15,16}$ Similar to the previous study of Le et $\mathrm{al},{ }^{12}$ we have included patients with small AVMs or DAVFs for whom the diagnosis of AVS remains challenging. In comparison with this previous study, we found a higher sensitivity of ASL for detecting AVS (95\% versus 78\%), which may be partly explained by the higher field strength we used, with the signal-to-noise ratio of the ASL sequence being markedly improved at 3T.

ASL/SWI provided a high diagnostic performance by detecting all the AVMs in our cohort and diagnosing significantly more patients with AVS in comparison with the conventional MR imaging protocol. While ASL is highly sensitive for detecting AVS, SWI appears more specific in providing anatomic details of the whole-brain vasculature and differentiating arteries from veins. Merged images between ASL and SWI also appeared particularly useful for precisely locating the ASL signal inside or outside a venous structure, further improving the detection of AVS. Most interesting, in our study, ASL/SWI correctly demonstrated AVS in 5 patients with small AVMs, while the conventional MR imaging protocol did not.

In patients with AVMs previously treated, contrast-enhanced MR images may be misleading. ${ }^{20}$ Specifically, dilated draining veins or enlarged arteries might persist in the absence of AVS because the decrease of vessel caliber after AVS occlusion often takes time to reverse. ${ }^{4}$ Likewise, contrast enhancement may occur in the treated nidus due to reactive gliosis. ${ }^{21}$ Our results suggest that ASL/SWI could be particularly effective in the specific setting of AVS under treatment. Indeed, the ASL fast spin-echo readout appears very suitable in patients with ICH or with previous embolization, for whom susceptibility artifacts are both common and widespread on MR images.

There are several limitations to our study. First, the diagnostic performance of SWI and/or ASL in case of slow-blood-flow AVS remains unclear, even if we investigated a large cohort of patients with various AVS. The high diagnostic performance of ASL/SWI in patients with treated AVMs, and thus with reduced blood-flow, suggests that this approach remains effective. Second, in our 
study, ASL was performed by using a postlabel delay of $1525 \mathrm{~ms}$, whereas it would have been useful to evaluate the accuracy of ASL by using shorter or higher values. The use of multiple postlabel delays may further improve locating the AVS. Further studies are required to test this hypothesis. Third, we did not evaluate the accuracy of ASL/SWI for the assessment of AVM or DAVF angioarchitecture. Indeed, we focused our study on the detection of AVS with MR imaging, considering that DSA will be systematically performed in all patients with a high suspicion of AVS. Finally, while a wide range of conditions such as hypervascular tumors, luxury perfusion, reperfusion, or seizures ${ }^{22}$ may induce ASL hyperintensity, we did not include patients with etiologies other than AVS.

\section{CONCLUSIONS}

Our study showed that the combined use of ASL and SWI may be an alternative to contrast-enhanced MRA for the detection of intracranial AVS. Special attention should also be given to hyperintense signal in venous structures on merged ASL/SWI because it appears highly correlated with AVS.

\section{ACKNOWLEDGMENTS}

We thank Rachid Mahdjoub, Guillermo Zanolli, and Bastien Perez from GE Healthcare for their support.

Disclosures: Ruben Tamazyan—UNRELATED: Travel/Accommodations/Meeting Expenses Unrelated to Activities Listed: Boehringer Ingelheim France, Comments: European School of Oncology Meeting in 2015. Michel Piotin-UNRELATED: Consultancy: Stryker, Medtronic, MicroVention, Balt, Penumbra*; Payment for Development of Educational Presentations: Stryker, Medtronic, MicroVention, Balt*. Raphael Blanc-UNRELATED: Payment for Development of Educational Presentations: Medtronic, MicroVention, Stryker.* *Money paid to the institution.

\section{REFERENCES}

1. Hofmeister C, Stapf C, Hartmann A, et al. Demographic, morphological, and clinical characteristics of 1289 patients with brain arteriovenous malformation. Stroke 2000;31:1307-10 CrossRef Medline

2. Gandhi D, Chen J, Pearl M, et al. Intracranial dural arteriovenous fistulas: classification, imaging findings, and treatment. AJNR AmJ Neuroradiol 2012;33:1007-13 CrossRef Medline

3. Alexander MD, Cooke DL, Nelson J, et al. Association between venous angioarchitectural features of sporadic brain arteriovenous malformations and intracranial hemorrhage. AJNR Am J Neuroradiol 2015;36:949-52 CrossRef Medline

4. Hadizadeh DR, von Falkenhausen M, Gieseke J, et al. Cerebral arteriovenous malformation: Spetzler-Martin classification at subsecond-temporal-resolution four-dimensional MR angiography compared with that at DSA. Radiology 2008;246:205-13 CrossRef Medline

5. Taschner CA, Gieseke J, Le Thuc V, et al. Intracranial arteriovenous malformation: time-resolved contrast-enhanced MR angiography with combination of parallel imaging, keyhole acquisition, and kspace sampling techniques at 1.5 T. Radiology 2008;246:871-79 CrossRef Medline

6. Nishimura S, Hirai T, Sasao A, et al. Evaluation of dural arteriovenous fistulas with $4 \mathrm{D}$ contrast-enhanced MR angiography at 3T. AJNR Am J Neuroradiol 2010;31:80-85 CrossRef Medline
7. Eddleman CS, Jeong HJ, Hurley MC, et al. 4D radial acquisition contrast-enhanced MR angiography and intracranial arteriovenous malformations: quickly approaching digital subtraction angiography. Stroke 2009;40:2749-53 CrossRef Medline

8. Hodel J, Blanc R, Rodallec M, et al. Susceptibility-weighted angiography for the detection of high-flow intracranial vascular lesions: preliminary study. Eur Radiol 2013;23:1122-30 CrossRef Medline

9. Jagadeesan BD, Cross DT 3rd, Delgado Almandoz JE, et al. Susceptibility-weighted imaging: a new tool in the diagnosis and evaluation of abnormalities of the vein of Galen in children. AJNR Am J Neuroradiol 2012;33:1747-51 CrossRef Medline

10. Jagadeesan BD, Delgado Almandoz JE, Moran CJ, et al. Accuracy of susceptibility-weighted imaging for the detection of arteriovenous shunting in vascular malformations of the brain. Stroke 2011;42: 87-92 CrossRef Medline

11. Letourneau-Guillon L, Krings T. Simultaneous arteriovenous shunting and venous congestion identification in dural arteriovenous fistulas using susceptibility-weighted imaging: initial experience. AJNR Am J Neuroradiol 2012;33:301-07 CrossRef Medline

12. Le TT, Fischbein NJ, André JB, et al. Identification of venous signal on arterial spin labeling improves diagnosis of dural arteriovenous fistulas and small arteriovenous malformations. AJNR Am J Neuroradiol 2012;33:61-68 CrossRef Medline

13. Wolf RL, Wang J, Detre JA, et al. Arteriovenous shunt visualization in arteriovenous malformations with arterial spin-labeling MR imaging. AJNR Am J Neuroradiol 2008;29:681-87 CrossRef Medline

14. Yu SL, Wang R, Wang R, et al. Accuracy of vessel-encoded pseudocontinuous arterial spin-labeling in identification of feeding arteries in patients with intracranial arteriovenous malformations. AJNR Am J Neuroradiol 2014;35:65-71 CrossRef Medline

15. Nabavizadeh SA, Edgar JC, Vossough A. Utility of susceptibilityweighted imaging and arterial spin perfusion imaging in pediatric brain arteriovenous shunting. Neuroradiology 2014;56:877-84 CrossRef Medline

16. Lüdemann L, Jedrzejewski G, Heidenreich J, et al. Perfusion imaging of cerebral arteriovenous malformations: a study comparing quantitative continuous arterial spin labeling and dynamic contrast-enhanced magnetic resonance imaging at 3 T. Magn Reson Imaging 2011;29:1157-64 CrossRef Medline

17. Ramalho J, Semelka RC, Ramalho M, et al. Gadolinium-based contrast agent accumulation and toxicity: an update. AJNR Am J Neuroradiol 2016;37:1192-98 CrossRef Medline

18. Adin ME, Kleinberg L, Vaidya D, et al. Hyperintense dentate nuclei on T1-weighted MRI: relation to repeat gadolinium administration. AJNR Am J Neuroradiol 2015;36:1859-65 CrossRef Medline

19. Kanda $\mathrm{T}$, Ishii $\mathrm{K}$, Kawaguchi $\mathrm{H}$, et al. High signal intensity in the dentate nucleus and globus pallidus on unenhanced T1-weighted MR images: relationship with increasing cumulative dose of a gadolinium-based contrast material. Radiology 2014;270:834-41 CrossRef Medline

20. Cashen TA, Carr JC, Shin W, et al. Intracranial time-resolved contrast-enhanced MR angiography at 3T. AJNR Am J Neuroradiol 2006;27:822-29 Medline

21. Yamamoto M, Ide M, Jimbo M, et al. Neuroimaging studies of postobliteration nidus changes in cerebral arteriovenous malformations treated by gamma knife radiosurgery. Surg Neurol 1996;45: 110-19; discussion 119-22 CrossRef Medline

22. Deibler AR, Pollock JM, Kraft RA, et al. Arterial spin-labeling in routine clinical practice, part 3: hyperperfusion patterns. AJNR Am J Neuroradiol 2008;29:1428-35 CrossRef Medline 